\title{
Reflection on the Contradiction Between Online and Physical Book Retailers in China —-Conceptions about Future Bookstore Design
}

\author{
Dongyang Chen ${ }^{1, *}$ \\ ${ }^{1}$ Entrepreneurship and Innovation, School of Management, University of San Francisco, \\ *Corresponding author. Email: dchen35@dons.usfca.edu
}

\begin{abstract}
The bookstore industry in China is facing a tremendous change today due to online retailers' participation which has encroached a significant portion of physical retailers' market shares. Hence, based on the increasingly severe competition, the paper aims at opportunities that may change the industry and projects associative conceptions about the future bookstore. In the sequence of demonstrations, the paper will first provide a macroscopical perspective to reveal today's arena of the bookstore industry and expound on different stakeholders' relationships. Besides, some paragraphs will mention scholars' constructive opinions within the industry dynamics. Subsequently, the paper will discuss the advancing operational activities and suggest specific trends or assumptions that will lead the future industrial ecosystem. In addition, topics that are slightly outside the industry will be correlated to corroborate that bookstores are expecting coalescence and innovation. In essence, the paper is dedicated to suggesting more possibilities for developing a feasible $\mathrm{O} 2 \mathrm{O}$ business model, serving as a guide for real-world bookstore operators.
\end{abstract}

Keywords: Future Bookstore, E-commerce, Physical Retailer, Profit Margin, O2O Business Model, Technology, Big Data, Positioning

\section{INTRODUCTION}

\subsection{Research Background}

The rapidly increasing e-commerce business has changed the bookstore industry in China in the past decade, and online retailers encroached a significant portion from traditional retailers, which leads to a more intricate status quo to the industry. However, with an accelerating competition, there is no signal indicating that China's bookstore industry has become more profitable than before. The IBISWorld Report for the bookstore industry points out that the annual growth of profit was only $0.4 \%$ compared to the $3.4 \%$ of annual revenue growth, showing a declining profit margin growth [1]. Hence, the new technology brings a further involution to bookstores rather than creating value for them, and such imbalance is the key to construct a new business model above the industry. Along with digitalization and other trends, it appears that a concatenation may fill up the gap between physical retailers and online retailers. When retailers often emphasize inner contradiction, the enterprise that first synthesizes traditional bookstores and online distribution may find a creative way to generate value.

An opportunity for bookstore operators is the low industry concentration since the major players only occupied about $56.3 \%$ market share in 2020, and most of them are online retailers. Chen analyzed that the market concentration of online bookstores is high, "with the top four online platforms accounting for over $93 \%$ of overall online book sales [1]." The offline concentration is relatively open to penetration, and the status quo can be changed if a proper $\mathrm{O} 2 \mathrm{O}$ (Online to Offline) business model can be implemented. Under this background, e-commerce companies like Amazon and Dangdang have attempted to open physical bookstores, and Dangdang has once come up with an ambitious objective to open 1,000 bookstores within three years [2]. While the online book-retailing market is becoming saturated, most major players realized that struggling with online distribution will only catalyse a zero-sum game. Thereupon, the query of how to innovate physical bookstores and coalesce electronic devices turns into an imperative topic. In the interview with Yan Wang, 
president of East China Normal University Press, an insightful perspective has been shared:

It also should not be extended just to the digital realm. The goal for a long-selling textbook or educational title should be to make use of the content and create a whole ecosystem around it that puts together the author, publisher, reader, and teacher for further collaboration and interaction. The ecosystem must also be enhanced with the application of new technologies and tools such as AI, analytics, Big Data, and social media to make it even more useful and relevant. It will enable the creation of new materialsancillaries, curated items, lectures, and audio or video content, for instance - that can enrich the repository. Then the content can be "hybridized" for multiformat and multichannel dissemination for a wider reach [3].

\subsection{Literature Review}

Meanwhile, Wang is not the only person who mentions the possibility of a "hybrid" bookstore, and Zhang has indicated that Big Data and cloud computing can expand customer value and book diversity. Moreover, by adopting the Internet of Things, "ecommerce and traditional bookstores need to be integrated with each other to provide services for customers [4]." In short, the combination between physical bookstores and e-commerce technology becomes an urgent objective to study, and the declining profit situation could be ceased by an ingenious synthesis that advents in the future.

Nevertheless, while most scholars have investigated the status quo of the bookstore industry and provided general directions to future operation, only a few have put forward a pragmatic scheme about future bookstore design. To be specific, even though bookstore operators have now realized that they need to combine online and offline activities, most of them still hesitate to implement new technologies into physical stores. Especially to some traditional bookstore operators, who recognize bookstore as a "literal sanctuary," it is hard for them to make changes since they resist impurity except for books. Conversely, the online retailers born in the digitalization time will sometimes neglect the muted demand from customers because their domain cares about economies of scale more than positioning strategy. The cognitive gap is what they need to stride over for better competitive edges to each side of players. Consequently, this paper would focus on constructing an applicable $\mathrm{O} 2 \mathrm{O}$ model for the future bookstore, balancing advantages for both traditional bookstore and e-commerce technology.

\subsection{Research Framework}

To a step-by-step future bookstore conception, the paper would first provide more details about industrial investigations, including but not limited to industry reports, academic papers, and official information. Then, after coming up with specific results, the paper would analyze the comparative data and customer portrait, leading to a less ambiguous discussion about how the future bookstore will like. Besides, relevant approaches to creating industrial value would be suggested. At last, a conclusion contains key findings, research significance, and study limitations would be given.

\section{METHOD}

The paper's research method is literature analysis, which mainly focuses on secondary sources. By adapting the data from industrial reports and official information, any assumption this paper generates is based on a concrete study from industry trends and comparative perspectives. The paper also cited several current academic papers that share important views that forecast industrial foreground, which support essential evidence to future bookstore conception. The above does not certify that the future bookstore that this paper mentioned will undoubtedly come true, yet it is likely that an industrial reformation is fermenting for all players.

In order to convey a comprehensive understanding, the paper would not only apply intra-industrial resources but also compare information outside of the industry. For instance, the coffee and restaurant industries would be analogized to strengthen the viewpoint that a new bookstore form will appear soon. Otherwise, the paper would extend the extant discussion about the $\mathrm{O} 2 \mathrm{O}$ model a bit to verify whether the synthesis is feasible or not and what kind of difficulties bookstore operators may encounter with the channels' collision.

\section{RESULTS}

\subsection{The Industrial Status Quo Based on PEST Analysis}

Primarily, though the bookstore industry has become more intricate than before, more underneath opportunities await exploration. To specify the vigorous signal of the industry, an elementary PEST (political, economic, social, and technological) analysis can reveal many positive signals.

\subsubsection{Political Aspect}

"In 2001, the government began inviting tenders for the distribution of educational books [1]." In the past, the Chinese government holds the only channel of selling educational books, and such exclusive distribution has taken a large portion of benefits from private bookstores. In China, educational books are the 
most popular category due to the vast number of undergraduates and graduates, and their market segmentation arrived at $44.6 \%$ in 2020 . Thus, the distribution's opening has provided more subsistence space for private bookstores and enhanced overall supply to the magnitude of students. Moreover, the government has allowed private bookstores to establish their chain stores in 2004, extending the upper limit of the industry considerably.

\subsubsection{Economic Aspect}

Although the bookstore industry's profit margin is declining, commercial real estate has shown a preference for this business, and many malls have now tried to collaborate with branded bookstores to increase customer retention. Take an example of Sisyphe, one of the largest chain bookstores in China; this company can acquire an incredibly cheap lease from commercial real estate. As is well known that most private bookstores fall before the high rent price, Sisyphe's chairman Jin told a journalist that he could often obtain a lease that is only $10 \%$ of the average price from malls, and in some cases, for free [5]. The reason is that malls are seeking more approaches to enhance cultural diversity and commercial activities. At present, the homogenization problem is increasingly severe in commercial real estate, and a unique bookstore can bring more visitors to malls, driving demand for restaurants and shopping.

\subsubsection{Social Aspect}

Urban areas are still the central battlefield for the bookstore industry, which occupied $74.3 \%$ of total revenue in 2020 [1]. In such regions, people often have higher disposable income and reading willingness, which also offers the proximity of transport to encourage reading behavior. Otherwise, bookstore operators have more pilot experiments in the urban area based on different levels of population density. Consequently, if a new bookstore form or a new $\mathrm{O} 2 \mathrm{O}$ model can be created, urban areas will likely be the first pilot place to witness its advent.

\subsubsection{Technological Aspect}

As mentioned above, online retailers have crashed traditional bookstores' livelihood, and the COVID-19 has accelerated this phenomenon. According to China's Book Retailing Market Report in 2020, online distribution for book retailing has arrived $79 \%$ now, and the most potent factor is the discount price [6]. However, many critics belittle this phenomenon by censuring that it will make "bad money drives out good," and such "more books for lower profit" situation will decrease the quality of books. E-commerce is undoubtedly the most influential technology that changed the book retailing market in the past decade, yet the means of adapting it could still be improved. Otherwise, the barbaric growth will plunder physical retailers and even the whole industry's market value.

Based on the above analysis, bookstore's stakeholders should have a multi-dimensional recognition of this industry, which suggests operators not regard bookstore as a simple trading floor. The bookstore industry represents substantial expectationsGovernment wants it to be a station of spreading knowledge, and commercial real estate wants it to draw up its cultural deposits. Besides, in a country like China with accelerating economics, intellectuals are yearning for more valuable books. Considering spiritual influence, the bookstore industry's impact on society is more than just an industry, and its advance should keep up with the times.

On the other hand, e-commerce has brought a new distribution to book retailing but suppressed physical bookstore's vivosphere, eventually leading to a zerosum game based on cost structure. Indeed, there will be one or two winners out of the arena. However, their profits are based on the accumulation of selling quantity, which is reached by sacrificing all other companies' profit margins. In the future, when traffic bonus is dispersed, "e-com providers will return to the nature of business - supply chain competition [7]." In brief, technology does not equal the relucent destination of the bookstore industry, and overload of the channel will compress industrial profit and reduce the overall quality of publishing books. To reconstruct the industrial balance and improve the operational environment, bookstores need repositioning in today's society.

\subsection{Introspection on Relationships between Customers and Retailers}

For a thorough understanding of the new bookstore conception, the key is still about creating value for bookstores' customers. Expressly, the matter does not limit who the primary consumers of physical bookstores are and who prefer purchasing books online; today's bookstore operators should focus on some common characteristics. Hence, the following paragraph will present readers' preferences reflecting on products segmentation:

Until December 2020, the most popular book categories were Educational Books and Culture and Education Books, which occupied $44.6 \%$ and $27.9 \%$ of all book sales. What followed the orders were Natural Science and Technology Books (7.9\%), Social Science and Philosophy Books (6.4\%), Children's Books (6.2\%), and Literature and Arts Books (5.0\%). The remaining $2 \%$ belongs to other relatively narrow categories [1]. Following the above data, educational materials are still the most favorable product to 
customers, which coincides with the situation that more students and intellectuals emerge in China. Thus, a new form of bookstore may concentrate on educational aspects and make a pilot trial on relevant areas like college. Nevertheless, more details of customer portraits for such places need identification.

Beyond customer segmentation, the core values that different distribution methods can bring audiences are salient. Therefore, in detail, the next paragraph will expound on the major strengths and weaknesses of physical bookstores and online bookstores:

\subsubsection{Physical Retailers}

For many readers, their prior choice of buying books is still to find a physical bookstore, and "bookstores providing premium services may attract more customers [1]." Moreover, the traditional bookstore can provide souvenirs and coffee for visitors, gaining extra revenue from transactions per customer. In addition, a physical site is more advantageous at developing a unique positioning, attracting customers to revisit and repurchase products. On the contrary, urban areas' high rent often annoy most operators, and online retailers' purchasing power makes it harder for private companies to get an inexpensive deal with publishers and suppliers. Thus, due to the online channel's traction, small but characteristic bookstores become no longer appealing to a significant part of readers.

\subsubsection{Online Retailers}

The core values that online channels can bring to customers are the limitless choices of book categories and the lucrative discount rate. E-commerce allows customers to select a book from an infinite long-tailed market, which ignores the physical bookshelf that disturbed bookstore operators. Besides, the low discount prices based on economies of scale are the primary reason the uptight family chose an e-commerce platform. However, the strengths are also the weaknesses. First, the online bookstore does not have a natural guide to serve customers, and its general filter usually makes readers overwhelmed by the "books sea." Also, a low discount rate will steal suppliers' and publishers' profits, which might be detrimental to a healthy ecosystem.

Neglecting the distribution methods, physical and online bookstores' customers are naturally the same. When people are purchasing books, they are acquiring particular knowledge that draws their interest. Subsequently, the only thing that has changed is the distribution channel, regardless of which sides' customers. Take a distant example of transportation, people in the 19th century commuted by train, and modern people drive their vehicles or use transportation App. Although there are different approaches, commuters' core demand is always the same - transport from one place to another. For books, what customers need is to access inner knowledge and adapt it to reality. What needs to be done is redefine the bookstore's role, but many players have not yet summed up the principles of playing the positioning.

The quandary to today's bookstore operators is dramatic, which comes with opportunities and constraints. When Dangdang made its ambitious objective of opening 1,000 physical bookstores in 2015, no one could foresee that its expansion plan would be stagnated in 2018 with no more than 200 offline stores and delisted from the public stock market [8]. Once upon a time, Dangdang was the most prominent online book retailer, but its recession seemed to declare that it is dangerous for online retailers to reach out to traditional bookstores and vice versa. Nevertheless, if such attempts were not given, the monopolistic dilemma would be more severe in today's bookstore industry.

For the new form of a bookstore, the deadlock between online and offline retailers must be solved, and the hinge is on whether a bookstore can connect the channel from online distribution to offline sites. In this paper's assumption, an innovative $\mathrm{O} 2 \mathrm{O}$ model should adapt physical stores as the primary carrier and energize them with e-commerce or internet technology, conveying more high-quality materials to society. Furthermore, by focusing on long-seller content, such a new bookstore should be able to "extract parts of it to create derivative products, adapt it to fit specific markets, or transform it to fit different platforms or distribution channels [3]."

Throughout the PEST analysis and distribution comparisons, the paper has demonstrated industrial conditions and customer attitude. The following section is about the pragmatic conception of the $\mathrm{O} 2 \mathrm{O}$ model and integrated bookstore. Within intra-industrial investigation and outside resources, a list of possible features about future bookstores will be given, yet such ideas are not equivalent to the complete picture of the future bookstore. The paper's train of thought is mainly for real operators' reference and a healthier ecosystem, which does not directly serve as an industrial solution to any situation.

\section{DISCUSSION}

Before dive deeper into the future bookstore picture, any business operator today should realize that ecommerce platforms have already become an inevitable choice for customers, no matter in books or other articles of daily use. Technically, such platforms are automatic optimization that draws customers closer to their final purchasing behavior. People no longer need a physical trolley to accommodate products, and what they need is to browse online and click the "add to cart" 
button. However, most people still purchase products like foods or shoes on physical sites, and reasons for that conclude but not limit to freshness, fitness, and linkage - such experience can be detached from an online purchase.

Consequently, bookstore operators must create a "necessary experience" for offline purchases that is irreplaceable. Many companies have operated activities that differentiate themselves from traditional bookstores, which neither correlate to pure e-commerce. Tsutaya (Culture Convenience Club) is the most successful representative of chain bookstores in Japan, yet this company announces that their business focus is on lifestyle contents, platform, and database [9]. From their operation, a scorecard system called "T-Card" has been established above their 70 million members, which can be shared with 175 thousand franchised outlets. Customers will obtain transferable credit each time they purchase in Tsutaya Bookstore. Such purchasing behavior or visit records will turn to information that can be utilized in their database. In detail, all the activities they developed are based on Big Data: Tsutaya would integrate and reanalyze with their gathered data, generating a specific investigation report for their business client. If clients have further requirements, they can even provide consultation services in content creation, building design, and shop decoration. By combining physical bookstores and data services, Tsutaya has constructed a strong capital inflow, which boosted their revenue from 1.8 to 3.2 billion USD from 2013 to 2019 [10].

Another innovative example would be Sisyphe in China, which owns more than 300 chain bookstores in operation. As mentioned, this company takes advantage of bonding with commercial real estate, and their average setup time for a new bookstore is only 45 days. In Sisyphe's positioning, they try to expand as much storefront space as possible based on reading materials, blending with cultural products and coffee bars to earn extra profits. Except for the functional zone, Sisyphe's bookstore has differentiated child and professional zones, filling up diversified customers' multi-demands [5]. Otherwise, Sisyphe has built its own logistics system, providing a better commodity flow to observe comparative data. Like Tsutaya, Sisyphe does not recognize itself as a general "bookseller" but an "experience provider," which offers them a broader space to compose demands.

There are many more cases like Tsutaya or Sisyphe attempting to expand customer value from the "bookseller" mindset, in a projection that diversification is the key to confronting online bookstores - which flatted and digitalized books' content. In the following paragraphs, the paper will accordingly introduce several trends or conceptions that are important to future bookstore design, illustrating the feasibility of combining physical bookstore and online distribution:

\subsection{Payment Method}

It is a common phenomenon in China now that people no longer bring wallets or cash because every payment can be made by phone check. Through WeChat or other apps, "easy, fast, and secure payment methods have also expanded the market for new business models such as online courses and audio-based languagelearning subscriptions [3]." The online payment method means more than just switching a way to check, which can expand new business models like delivery service, offline takeaway, and subscription plan. In addition, the physical bookstore can create a deeper relationship with their customer by presenting customized services, establishing a complete membership system.

\subsection{Big Data}

Database is a vital component of the e-commerce business, and its function has been well explored in many industries in the past decade. There are many things an enterprise can accomplish with data, including but not limit to customer analysis, recommended algorithm, and transaction improvement. For instance, a bookstore can build a customer-oriented platform based on the $\mathrm{O} 2 \mathrm{O}$ model, adapting different data to classify book categories better and improve purchasing experience. "By using of large data and other related technologies, the platform will establish the business analysis model to optimize business decision-making and provide personalized information push service [4]." When enough information is installed into a database, bookstores like Tsutaya can use it to explore plenty of business opportunities.

\subsection{Network Effect}

Bookstore operators need to know the rule of today's arena, which is to utilize the internet influence. There are many different traction ways to get in touch with customers, including but not limited to the newspaper, television, social media, and video games. Based on disparate account operations, a company can establish a three-dimensional image to connect potential customers. "Typically, the greater the number of buyers, sellers, or users, the greater the network effect - and the greater the value created by offering [11]." If a bookstore can build a consistent bond between its physical sites and online information, the overlay of specific images will be magnified and generate strong traction. 


\subsection{Lifestyle Bookstore}

It is a model that many famous bookstores have applied now, which expanded their product line to more than just reading materials. As Muneaki Masuda, the CEO of Culture Convenience Club told a journalist that "the bookstore's problem is that it only sells books." In Tsutaya, what beside musical magazines are numerous DVDs and CDs; food books are in adjoin with finest kitchen wares; healthy books are besides well-designed treadmills. Moreover, beer lovers can even find British craft beer BrewDog IPA besides books about beer culture history [12]. There are many more possibilities around such examples, and the key for bookstore operators is to provide a memorable experience for their customers rather than just selling books.

\subsection{Cross-industrial Coalescence}

As mentioned, some bookstores now would collaborate with malls for lower rent, but there could be more choices when selecting a commercial partner. For example, a bookstore can aim at education and cooperate with colleges, providing relative resources for students and strengthening its market positioning. Furthermore, coffee bars or restaurants can be a partner, which may help a bookstore attract relevant customers and regurgitate client engagement to feedback. Specifically, a bookstore can narrow down its positioning to draw customer attention, which also decides the potential industry and company that will be the collaborator. The disadvantage is that a bookstore could mistakenly predict the niche market size, which constrains its future development.

\subsection{Miniature Bookstore}

This is a theoretical conception of the future bookstore, which only represents the paper's limited projection, indicating that the future bookstore could be far smaller than the present size. Imagine a bookstore as small as a telephone booth or convenience store, and there are no books inside it except for an elaborate book printer-customers can directly print the book they checked out on a mobile app. On the above of that, if the customer has a certain extent of freedom to customize the printed material, the purchasing experience will be far more straightforward and novel. This idea comes with the current trend of some coffee bars and restaurants shrinking their store size, which ingratiates them with increasingly more "grab and go" customers. Regarding the consumption trend, McDonald's China CEO Phyllis Cheung says that "restaurant formats can be smaller because people are looking for fast service; white-collar workers in the central business district have more incidence of 'grab and go' [13]." It appears in a similar case that the new café brand Manner started attracting capital, which acquired 1.3 billion USD appraisement in just a few years. Manner's uniqueness compares to traditional café is that they barely provide seats in the store, and most of their stores are smaller than 5 square meters [14]. Undoubtedly, the bookstore is quite a different industry to the above examples, yet modern consumers' fast rhythm does show the intent of yearning for faster service.

In summary, bookstore operators should expand their industrial map to seek opportunities, establish their unique strengths on specific domains, and build a proprietary moat above crucial values. The most helpful instruction for the future bookstore is that "do not just sell books," which indicates that extra values like concentration, culture, and lifestyle are expected from customers. As "The Third Space" concept that Starbucks CEO Kevin Johnson reiterated in his internal letter that "Starbucks is committed to create a thirdplace experience that is exactly tuned to that need providing safe, familiar, and convenient experiences [15];" what beyond the product is the meaning underneath it. In order to construct an effective $\mathrm{O} 2 \mathrm{O}$ business model that complies with today's society, bookstores need not only to know how to seize advantages from technology usage but also develop their unique positioning toward customers.

\section{CONCLUSION}

\subsection{Key Findings}

Throughout the paper, the industrial status quo and stakeholders' relationship provided a more in-depth consideration of the bookstore's future potential, encouraging that online and physical retailers should coexist with each other. Besides, the paper suggested that today's bookstore operators should learn how to construct an $\mathrm{O} 2 \mathrm{O}$ business model that accommodates various possibilities, taking advantage of political, economic, social, and technological aspects.

Furthermore, the paper projected six significant industrial insights that could have changed bookstores' arena, including payment method, big data, network effect, lifestyle bookstore, cross-industrial coalescence, and miniature bookstore. However, more possibilities await explorations since books are a carrier of miscellaneous contents, which would not be constrained by particular topics, cultures, and perspectives.

\subsection{Research Significance and Limitations}

As the introduction mentioned, today's bookstore industry faces a declining profit margin and increasing competition; the more intricate circumstance has overwhelmed many private bookstores. In such a period, private bookstores must invent an innovation that can deter industrial involution, finding a new way to create 
value for the whole ecosystem. Otherwise, a more rigid online hierarchy may reap most players' benefits and eventually lead to a stagnant environment that harms retailers, suppliers, and customers.

Consequently, the paper reflected bookstores' bottlenecks and offered suggestions that may unfasten the deadlock, providing more pragmatic conceptions about future bookstore design. Although unfortunately, the paper is not an immediate solution to most industrial quandaries, it may inspire those operators who want to build an $\mathrm{O} 2 \mathrm{O}$ business model and redefine their functional positioning. Moreover, the paper is dedicated to promoting a more diversified bookstore ecosystem, which may enrich bookstores' forms and create value for stakeholders.

Regarding the research limitations, the paper has mainly used secondary data to demonstrate key findings, lacking primary data and timely observation. Otherwise, there are insufficient descriptions of some vital components like publishers, used books market, and technological accessibility; such deficiencies may cause incomprehensive understanding of some operational conditions. Therefore, bookstore operators should recognize the paper as a reference while adapting it to reality.

\section{REFERENCES}

[1] IBISWorld. (2020). Bookstores in China. In IBISWorld Industry Research Reports in China. IBISWorld.

[2] Liu, Z. (2018). Whither the Book Retailing Industry in China: A Historical Reflection. Publishing Research Quarterly, 34(1), 133. https://doi.org/10.1007/s12109-018-9569-0

[3] Tan, T. (2019, September 30). Checking of the Academic Book Market in China: China's academic book segment is flourishing amid market shifts and technological advances. Publishers Weekly, 266(39), 35.

[4] Zhang, J. (2017). The Integration of Technology and the Publishing Industry in China. Publishing Research Quarterly, 33(2), 173-182. https://doi.org/10.1007/s12109-017-9504-9

[5] Yang, Q. (2014, October 24). How did Sisyphe "Roll the Rock" to the Whole Nation? Tencent Finance. https://finance.qq.com/a/20141024/004558.htm

[6] People's Daily. (2021). China's Book Retailing Market Report in 2020: Risks and Variations on Book Market. Xinhuanet. http://www.xinhuanet.com/culturepro/202101/29/c_139703495.htm
[7] Qian, W., Wu D. (2016). Re-thinking on the Path of Traditional Publishing Reform. Beijing: China, vol. 22.

[8] He, S. (2020, April 7). Dangdang Continuously Closed Offline Bookstores, Encountering an Expansion Challenge. MarketWorld. https://www.163.com/dy/article/F9KPD55T0519S UH3.html

[9] T-site Investment (Shanghai) Co.. (2021, April 6). Brand Concept. https://www.cccchn.com/qiyelinian/

[10] Zhao, H. (2020, December 23). T-site is Opening in Shanghai but It is not Designed to Sell Books. YiMagazine.

https://www.yicai.com/news/100887816.html

[11] Stobierski, T. (2020, November 12). What Are Network Effects? Harvard Business School Online. https://online.hbs.edu/blog/post/what-are-networkeffects

[12] Liu, F. (2019, October 9). Tsutaya Bookstore and Its Product Thinking. 36Kr. https://www.36kr.com/p/1724475752449

[13] McKinsey \& Company. (2021). Leading through a Crisis: How McDonald's China CEO Phyllis Cheung Re-ignited Growth during COVID-19. In China Consumer Report 2021. McKinsey \& Company.

[14] Food \& Beverage Innovation Forum. (2021, March 15). Boutique Coffee Brand Manner Has Raised a New Round of Funding at a Valuation of $\$ 1.3$ Billion. Food \& Beverage Innovation Forum. https://www.163.com/dy/article/G4AOR5T90519F EEC.html.

[15] Starbucks Coffee Co.. (2020, May 4). Starbucks Ceo: The Third Place, Needed Now More than Ever Before. https://stories.starbucks.com/press/2020/starbucksceo-the-third-place-needed-now-more-than-everbefore. 